\title{
Composição química e perfil de ácidos graxos do leite e muçarela de búfalas alimentadas com diferentes fontes de lipídeos
}

\author{
[Chemical composition and fatty acids profile in milk and mozzarella cheese of \\ water buffalo fed different lipid sources] \\ R.L. Oliveira ${ }^{1}$, M.M. Ladeira ${ }^{2}$, M.A.A.F. Barbosa ${ }^{3}$, M. Matsushita ${ }_{5}^{4}$, G.T. Santos ${ }^{4,6}$, \\ A.R. Bagaldo ${ }^{1,7}$, R.L. Oliveira ${ }^{5}$ \\ ${ }^{1}$ Escola de Medicina Veterinária - UFBA \\ Av. Ademar de Barros, 500 \\ 40170-110 - Salvador, BA \\ ${ }^{2}$ Universidade Federal de Lavras - Lavras, MG \\ ${ }^{3}$ Universidade Estadual de Londrina - Londrina, PR \\ ${ }^{4}$ Universidade Estadual de Maringá - Maringá, PR \\ ${ }^{5}$ Aluna de graduação - UPIS - Brasília, DF \\ ${ }^{6}$ Bolsista do $\mathrm{CNPq}$ \\ ${ }^{7}$ Bolsista FABESP
}

\section{RESUMO}

Avaliaram-se a composição química e o perfil de ácidos na gordura do leite de búfalas alimentadas com fontes de lipídeos. Foram utilizadas nove búfalas em lactação, multíparas e com produção média diária de leite de $6,5 \mathrm{~kg}$. O delineamento experimental foi quadrado latino $3 \times 3$, triplo, composto por três tratamentos, três períodos de 21 dias e nove repetições. Os tratamentos foram dietas sem lipídeo adicional, com grão de soja e com óleo de soja. A proporção de silagem de milho variou entre 70 e $75 \%$. A inclusão de óleo de soja elevou os teores de gordura no leite e no queijo tipo muçarela. As concentrações de ácidos graxos saturados (AGS) variaram de 62,8 a $69,8 \%$. Não houve diferenças nos teores de AGS entre os tratamentos sem lipídeo adicional e com grão de soja. O tratamento com óleo de soja resultou em queda de $10 \%$ no teor de AGS. As fontes lipídicas reduziram as concentrações de AGS e aumentaram as concentrações de ácidos graxos insaturados. Ácidos graxos encontrados na muçarela, em ordem decrescente, foram: palmítico, oleico, láurico e esteárico. O óleo de soja apresentou maior capacidade de elevar as concentrações do ácido linoleico conjugado (CLA) e do ácido vaccênico no leite e no queijo muçarela.

Palavras-chave: ácido linoleico conjugado, bubalinos, grão de soja, lactação, óleo de soja

\begin{abstract}
The chemical composition of milk and fatty acids profile in milk fat of water buffaloes fed different lipid sources were evaluated. Nine lactating multiparous water buffaloes, averaging $6.5 \mathrm{~kg}$ of milk daily, were used. The statistical design was $3 \times 3$ triple Latin Square, three periods of 21 days and nine repetitions. The treatments were diet without additional fat, diet with soybean grain, and diet with soybean oil. Corn silage content in the diets ranged from 70 to $75 \%$. The inclusion of soybean oil increased fat content in milk and mozzarella. Saturated fatty acid (SFA) concentrations in milk ranged from 62.8 to 69.8\%. SFA content was similar in milk for the diets without additional fat and with soybean grain. However, in the diet with soybean oil, SFA decreased about 10\%. Lipid sources reduced SFA and increased unsaturated fatty acids (UFA). The main fatty acids found in mozzarella fat, in decreasing array, were: palmitic, oleic, lauric, and estearic. Diet with soybean oil presented higher capacity of increasing conjugated linoleic acid (CLA) and vaccenic acid concentrations in milk and mozzarella.
\end{abstract}

Keywords: buffaloes, conjugated linoleic acid, lactation, soybean grain, soybean oil

Recebido em 9 de julho de 2008

Aceito em 5 de fevereiro de 2009

Email: ronaldooliveira@ufba.br 


\section{INTRODUÇÃO}

O leite de búfala é, do ponto de vista industrial, de melhor qualidade do que o leite de vaca. Isto ocorre devido às maiores concentrações em sólidos totais, gordura, proteína, cálcio e fósforo (Dubey et al., 1997; Patel e Mistry, 1997), o que leva ao maior rendimento na produção de derivados, como: queijo, manteiga, leite em pó e outros produtos fermentados. Dentre os derivados oriundos do leite de búfala, destaca-se a produção do queijo tipo muçarela.

Entre os componentes do leite de búfala, em relação ao leite de vaca, a gordura apresenta grandes diferenças de concentrações, que variam de 6,5 a 7,9\% (Nader Filho et al., 1984; Macedo et al., 2001).

Atualmente, o consumidor possui grande preocupação com a quantidade de gordura nos alimentos, pois este associa a ingestão de gordura ao risco de doenças cardiovasculares. As gorduras que contêm ácidos graxos saturados (AGS), em geral, elevam os níveis de LDL no sangue humano, quando comparadas com proteínas, carboidratos ou ácidos graxos insaturados em substituições isoenergéticas. Todavia, o efeito hipercolesterolêmico dos AGS está associado apenas aos ácidos láurico, mirístico e palmítico (Grummer, 1991; Farfan, 1996). Portanto, é importante que se tenha conhecimento do perfil de ácidos graxos da gordura do leite de búfala e sua possível influência sobre a saúde humana.

Polidori et al. (1997) verificaram que o leite de búfala continha aproximadamente $33 \%$ de ácidos graxos insaturados (AGINS), ou seja, 67\% de AGS. Ao considerar os teores dos AGS prejudiciais à saúde humana, os valores descritos por Polidori et al. (1997) foram semelhantes aos encontrados por Oliveira et al. (2007) para os ácidos láurico e mirístico, no leite de vaca. Contudo, para o ácido palmítico, os valores encontrados foram, respectivamente, 32 e $26 \%$, ou seja, o leite de búfala apresentou seis pontos percentuais a mais que o leite de vaca.

Quando se estuda a gordura no leite de búfala, além do perfil de ácidos graxos, torna-se importante avaliar a concentração do ácido graxo linoleico conjugado, ou CLA. Sua importância se deve a investigações com animais e modelos celulares que estabeleceram que o CLA apresenta atividade anticarcinogênica para vários tipos de tumores (Bauman et al., 2004). Dentre os alimentos que apresentam maior quantidade deste ácido graxo estão aqueles de origem de ruminantes (leite, carnes), sendo que o principal CLA encontrado, 80 a 90\%, é o isômero C18:2 cis-9, trans-11.

Este experimento teve como objetivo avaliar os efeitos da inclusão de duas fontes de lipídeos, o óleo e o grão de soja, na alimentação de búfalas lactantes sobre a composição química e o perfil de ácidos graxos no leite e na muçarela.

\section{MATERIAL E MÉTODOS}

Nove búfalas em lactação, multíparas, com peso vivo $(\mathrm{PV})$ médio de $683 \pm 17 \mathrm{~kg}$ e produção média diária de leite de $6,5 \mathrm{~kg}$ foram utilizadas, no período de setembro a dezembro de 2003.

Antes do experimento os animais foram pesados, vermifugados e distribuídos aleatoriamente em três dietas com diferentes fontes de lipídeos: dieta sem lipídeo adicional ou dieta controle; dieta com grão de soja, dieta com óleo de soja. A proporção de volumoso das dietas variou entre 70 e $75 \%$, constituído de silagem de milho. Utilizou-se o delineamento experimental em quadrado latino $3 \times 3$, triplo, composto por três tratamentos, três períodos e nove repetições. O experimento teve duração de 63 dias, compostos por períodos de 21 dias, sendo 14 para a adaptação dos animais às dietas experimentais e sete para coleta de amostras. A proporção percentual dos componentes das dietas experimentais e a composição bromatológica encontram-se nas Tab. 1 e 2.

Os animais foram alojados, individualmente, em baias de $24 \mathrm{~m}^{2}(3 \times 8 \mathrm{~m})$ cada. A dieta foi fornecida à vontade, na forma de mistura completa, duas vezes ao dia. Água foi fornecida à vontade. Os animais foram alimentados logo após as ordenhas das 5 e 16h. As sobras foram pesadas diariamente, e o fornecimento foi ajustado de maneira que a proporção das mesmas ficasse entre $10 \%$ e $20 \%$. Amostras dos alimentos foram coletadas para as análises laboratoriais. 
Tabela 1. Composição químicobromatológica dos ingredientes das dietas para búfalas lactantes

\begin{tabular}{|c|c|c|c|c|c|c|c|}
\hline \multirow{2}{*}{ Componentes } & \multirow{2}{*}{$\begin{array}{l}\text { MS } \\
(\%)\end{array}$} & \multicolumn{6}{|c|}{$\%$ da matéria seca } \\
\hline & & $\mathrm{MO}$ & PB & FDN & FDA & $\mathrm{EE}$ & CNF \\
\hline Silagem de milho & 32,74 & 92,20 & 7,88 & 52,63 & 34,34 & 2,96 & 28,73 \\
\hline Milho grão moído & 89,30 & 98,98 & 8,42 & 10,56 & 15,05 & 3,61 & 76,38 \\
\hline Farelo de soja & 90,62 & 94,02 & 45,73 & 13,46 & 9,93 & 0,82 & 34,00 \\
\hline Óleo de soja & 100,00 & 100,00 & 0,00 & 0,00 & 0,00 & 100,00 & 0,00 \\
\hline Grão de soja & 91,12 & 95,54 & 40,46 & 24,53 & 28,16 & 15,70 & 14,85 \\
\hline
\end{tabular}

MS: matéria seca; MO: matéria orgânica; PB: proteína bruta; FDN: fibra em detergente neutro; FDA: fibra em detergente ácido; EE: extrato etéreo; CNF: carboidratos não-fibrosos.

Tabela 2. Proporção percentual dos componentes nas dietas experimentais e composição bromatológica, com base na matéria seca das dietas oferecidas às búfalas lactantes

\begin{tabular}{lccc}
\hline Componentes & $\begin{array}{c}\text { Sem lipídeo } \\
\text { adicional }\end{array}$ & $\begin{array}{c}\text { Grão de } \\
\text { soja }\end{array}$ & $\begin{array}{c}\text { Óleo de } \\
\text { soja }\end{array}$ \\
\hline Silagem de milho & 70,00 & 75,00 & 74,72 \\
Milho grão moído & 15,94 & 8,58 & 8,33 \\
Farelo de soja & 12,03 & 1,89 & 12,70 \\
Grão de soja & --- & 12,50 & --- \\
Óleo de soja & --- & --- & 2,21 \\
Calcário & 0,31 & 0,31 & 0,31 \\
Fosfato bicálcico & 1,72 & 1,72 & 1,72 \\
\hline & Composição bromatológica (\%MS) \\
\hline Proteína bruta & 11,89 & 12,05 & 11,96 \\
Fibra em detergente neutro & 41,40 & 44,60 & 42,72 \\
Fibra em detergente ácido & 28,29 & 31,09 & 28,61 \\
Extrato etéreo & 2,76 & 4,34 & 4,85 \\
Carbohidratos não-fibrosos & 35,45 & 30,28 & 31,68 \\
Cinzas & 8,51 & 8,73 & 8,79 \\
\hline
\end{tabular}

Durante a fase de coleta, em cada período experimental, foram colhidas, diariamente, amostras dos alimentos fornecidos e das sobras. As amostras foram pré-secas em estufa com ventilação forçada a $55^{\circ} \mathrm{C}$ por $72 \mathrm{~h}$. Após a présecagem, as amostras foram moídas em peneira com crivos de $1 \mathrm{~mm}$ e homogeneizadas, originando amostras compostas por período e por animal. Análises de matéria seca (MS), proteína bruta (PB), extrato etéreo (EE), fibra em detergente ácido (FDA) e cinzas foram realizadas segundo metodologia descrita no AOAC (Official..., 1990), e de fibra em detergente neutro (FDN), segundo Van Soest et al. (1991). Os teores de CNF foram calculados segundo Mertens (1987), em que $\mathrm{CNF}=100-$ (FDN+PB+EE+cinzas).

Nos sete dias de coleta de cada período experimental, foram medidas as produções de leite em $\mathrm{kg} /$ dia, que foram também ajustadas para $4 \%$ de gordura por meio da equação proposta por Gaines e Davidson (1925). Quatro $\mathrm{kg}$ de leite por dia foram coletados e armazenados $\mathrm{a}-20^{\circ} \mathrm{C}$ para produção da muçarela.

O CLA e os ácidos graxos das amostras de leite e muçarela foram determinados. As análises foram feitas por meio da transesterificação dos ácidos graxos pela metodologia descrita pela ISO (Animal..., 1978) com o emprego de solução de nheptano e $\mathrm{KOH} / \mathrm{metanol}$. Os ésteres de ácidos graxos foram quantificados por cromatografia gasosa, equipado com detector de ionização de chama e coluna capilar de sílica fundida com $100 \mathrm{~m}$ de comprimento, $0,25 \mathrm{~mm}$ de diâmetro interno e 0,20 $\mu \mathrm{m}$ de Carbowax 20M; com fluxo de $1,2 \mathrm{~mL} / \mathrm{min}$ de $\mathrm{H}_{2}$ (gás de arraste), $30 \mathrm{~mL} / \mathrm{min}$ de $\mathrm{N}_{2}$ (gás auxiliar), 30 e $300 \mathrm{~mL} / \mathrm{min}$, para $\mathrm{o}_{2}$ e ar sintético para a chama do detector. A temperatura 
inicial da coluna foi estabelecida em $150^{\circ} \mathrm{C}$, mantida por $3 \mathrm{~min}$, e progressivamente aumentada até $240^{\circ} \mathrm{C}$, com taxa de elevação de $10^{\circ} \mathrm{C} / \mathrm{min}$. O perfil de ácidos graxos foi expresso em porcentagem do total de ácido graxo padrão, que tem valores certificados para 11 ácidos graxos. Estes são usados para estabelecer os fatores de correção para cada um dos ácidos graxos certificados que foram usados para transformar o pico em área para peso (mg/g ácidos graxos totais).

A produção de leite inicial de cada búfala foi utilizada como covariável. Os dados foram submetidos à análise de variância e as médias comparadas pelo teste Tukey a $5 \%$ de probabilidade, utilizando-se o programa de SAEG (Sistema..., 1997).

\section{RESULTADOS E DISCUSSÃO}

Os teores de sólidos totais e proteína bruta do leite e da muçarela não foram influenciados $(\mathrm{P}>0,05)$ quando as búfalas foram alimentadas com as diferentes fontes de lipídeos na dieta (Tab. 3). A média geral para o teor de sólidos totais foi de $19,8 \%$, valor acima do encontrado na literatura, que variam de $17 \%$ (Verruma e Salgado, 1994) até 18,1\% (Amaral et al., 2004). $\mathrm{O}$ teor médio da proteína bruta no leite $(5,9 \%)$ pode ter contribuído para esse valor de sólidos totais, pois também estão acima dos encontrados por Verruma e Salgado (1994), Macedo et al. (2001) e Amaral et al. (2005). Segundo Amaral et al. (2005), fatores ambientais, como estação de ano e nutrição, podem afetar a composição do leite de búfalas. Nesse contexto, como as búfalas eram mantidas individualmente e dispunham de alimentos à vontade, esse manejo permitiu incremento na composição do leite, comparado com as respostas observadas pelos autores em animais em sistema extensivo. Andriguetto et al. (2005) encontraram valores semelhantes para o teor de proteína bruta, 5,63\%, no leite de búfalas alimentadas com dietas à base de capim-elefante e silagem de sorgo, em sistema de manejo similar ao do presente trabalho.

Tabela 3. Composição do leite e da muçarela de búfalas alimentadas com diferentes fontes de lipídeos na dieta (\%MS)

\begin{tabular}{|c|c|c|c|c|}
\hline Componentes & Sem lipídeo adicional & $\begin{array}{c}\text { Grão de } \\
\text { soja }\end{array}$ & $\begin{array}{c}\text { Óleo de } \\
\text { soja }\end{array}$ & CV $(\%)$ \\
\hline \multicolumn{5}{|c|}{ Leite } \\
\hline Sólidos totais & 20,12 & 20,94 & 18,30 & 26,12 \\
\hline Proteína bruta & 5,97 & 6,30 & 5,50 & 13,14 \\
\hline Gordura & $8,66 \mathrm{~b}$ & $8,95 b$ & $9,40 \mathrm{a}$ & 18,21 \\
\hline \multicolumn{5}{|c|}{ Muçarela } \\
\hline Sólidos totais & 42,38 & 46,43 & 44,84 & 22,25 \\
\hline Proteína bruta & 42,59 & 45,41 & 42,82 & 21,13 \\
\hline Gordura & $21,05 \mathrm{~b}$ & $21,75 b$ & $25,27 \mathrm{a}$ & 11,27 \\
\hline
\end{tabular}

Médias, na mesma linha, seguidas de letras distintas diferem entre si, pelo teste Tukey $(\mathrm{P}<0,05)$.

$\mathrm{CV}$ : coeficiente de variação.

A inclusão de óleo de soja na dieta elevou o teor de gordura $(\mathrm{P}<0,05)$, tanto no leite, quanto na muçarela. Estudos com a utilização de lipídeos na dieta de búfalas lactantes são escassos. Entretanto, trabalhos com alimentação de fontes lipídicas para vacas leiteiras têm constatado respostas diferentes. Rabello et al. (1996) e Vargas et al. (2002) não verificaram diferenças na composição de leite de vacas alimentadas com grão ou óleo de oleaginosas, em até $7 \%$ de extrato etéreo na dieta. Enquanto Oliveira et al. (2007) relataram redução no teor de gordura no leite de vacas alimentadas com fontes de lipídeos.
Normalmente, a utilização de lipídeos na dieta de ruminantes resulta em queda nos teores de gordura do leite (Bauman e Griinari, 2003). A teoria mais aceita baseia-se no princípio de que certas condições nutricionais, como o teor de lipídeo e o $\mathrm{pH}$ ruminal, afetam a bio-hidrogenação ruminal dos ácidos graxos, levando à síntese de ácidos graxos Trans, que são potentes inibidores da síntese de novo na glândula mamária. Baumgard et al. (2001) observaram que a infusão de Trans-10 cis-12 no abomaso reduziu em $44 \%$ a percentagem de gordura do leite. Provavelmente a alta quantidade de volumoso na dieta não permitiu que ocorressem grandes alterações na bio-hidrogenação ruminal e, 
consequentemente, a queda na gordura no leite. Além disso, os teores de extrato etéreo nas dietas com óleo de soja estão abaixo dos limites máximos preconizados para alimentação de ruminantes, que são entre 6 a 7\% (Nutrient..., 2001).

As concentrações de AGS variaram de 62,8 a $69,8 \%$ (Tab. 4). Não houve diferença $(\mathrm{P}>0,05)$ nos teores desses ácidos graxos entre as dietas sem lipídeo adicional e com grão de soja. O tratamento com óleo de soja resultou em queda aproximada de $10 \%(\mathrm{P}<0,05)$ em relação aos outros tratamentos. As concentrações dos AGMI foram 23\% maiores na dieta com óleo de soja em relação à dieta sem lipídeo adicional. $\mathrm{O}$ tratamento com grão de soja diferiu da dieta sem lipídeo adicional $(\mathrm{P}<0,05)$ quanto à concentração dos APINS.

Polidori et al. (1997) verificaram que o leite de búfala continha aproximadamente $33 \%$ de AGINS, ou seja, $67 \%$ de AGS, valor similar aos observados nas dietas sem lipídeo adicional e com grão de soja. Van Nieuwenhove et al. (2004), ao trabalharem com búfalas alimentadas com pastagem natural, encontraram valores de 59\% para os AGS de cadeia longa.

Ao se considerar apenas os AGS capazes de elevar os níveis de LDL sanguíneo (láurico, palmítico e esteárico), pode-se observar que as dietas com fontes de lipídeos reduziram suas concentrações $(\mathrm{P}<0,05)$, com destaque para a dieta com óleo de soja. Os valores totais dos três AGS encontrados foram 45,8; 43,9 e 43,0\%, respectivamente, para os tratamentos, sem lipídeo adicional, com grão de soja e com óleo de soja. Com isso, houve aumento nos AG com mais de 18 carbonos na dieta com óleo de soja.

Grummer (1991) descreveu que a inclusão de óleo de canola e de girassol na dieta de vacas em lactação pode reduzir a concentração de ácidos graxos de cadeia curta e média $\left(\mathrm{C}_{4: 0}\right.$ a $\left.\mathrm{C}_{12: 0}\right)$ de 20 a $40 \%$ e aumentar de 55 a $80 \%$ a percentagem de ácidos graxos de cadeia longa $\left(\mathrm{C}_{18: 0}\right.$ e $\left.\mathrm{C}_{18: 1}\right)$. Griinari et al. (2000) também encontraram redução de $32 \%$ na concentração de ácidos graxos de cadeia curta e média $\left(\mathrm{C}_{4: 0}\right.$ a $\left.\mathrm{C}_{12: 0}\right)$ na gordura do leite de vacas alimentadas com dietas ricas em concentrado e ácidos graxos insaturados livres.

Os ácidos graxos da gordura do leite têm duas origens: a síntese de novo e os ácidos graxos da corrente sanguínea (origem dietética, 88\%; ou endógena, 12\%). Os AG com até 14 carbonos são formados a partir da síntese de novo, e o ácido palmítico é oriundo dos dois mecanismos, cada um desses representa aproximadamente 50\% (Grummer, 1991). Segundo Palmquist e Jenkins (1980), o possível efeito dos AGINS na dieta de ruminantes seria sobre a síntese de novo da gordura do leite, já que haveria redução na atividade da enzima acetil-CoA carboxilase. Isso explica a queda nos AG hipercolesterolêmicos observada nesta pesquisa.

O teor de CLA (C18:2, cis 9, trans 11) no leite das búfalas foi influenciado pelas fontes de lipídeos nas dietas $(\mathrm{P}<0,05)$; no tratamento com óleo de soja, os teores de CLA praticamente dobraram. Estes valores estão na mesma faixa dos encontrados por Jiang et al. (1996), que relataram variação de 0,25 a $1,8 \%$ de CLA na gordura do leite de vacas. Van Nieuwenhove et al. (2004) observaram teor de $0,48 \%$ de CLA no leite de búfalas, valor este bem abaixo das três dietas avaliadas neste estudo.

Dhiman et al. (2000) verificaram aumento de $237 \%$ na produção de CLA em vacas em lactação que receberam $2 \%$ de óleo de soja na dieta. No Brasil, Eifert et al. (2006) observaram que a inclusão de óleo de soja na dieta de vacas proporcionou aumento de $230 \%$ no conteúdo de CLA.

Segundo Oliveira et al. (2007), a concentração de CLA nos produtos derivados dos ruminantes depende de dois processos: a bio-hidrogenação ruminal e a dessaturação endógena do ácido vaccênico pela $\Delta^{9}$-dessaturase nos tecidos; em que a dieta é o principal fator que influencia os teores de CLA.

$\mathrm{Na}$ Tab. 5, apresenta-se o perfil de ácidos graxos da gordura do queijo muçarela. No geral, o perfil de AG no leite e no queijo foi semelhante. Resultados similares foram encontrados por Fox et al. (1993) e Dhiman et al. (1999). As fontes lipídicas reduziram as concentrações de AGS e aumentaram as de AGINS $(\mathrm{P}<0,05)$, o que fez a relação AGINS/AGS variar de 0,40 a 0,56.

Os principais ácidos graxos encontrados na gordura da muçarela, em ordem decrescente, foram: ácido palmítico, oleico, láurico e esteárico. A mesma ordem foi encontrada por Van Nieuwenhove et al. (2004). 
Composição química e perfil de ácidos...

Tabela 4. Ácidos graxos da gordura do leite de búfalas alimentadas com diferentes fontes de lipídeos na dieta

\begin{tabular}{|c|c|c|c|c|}
\hline Ácido graxo & $\begin{array}{c}\text { Sem lipídeo } \\
\text { adicional }\end{array}$ & $\begin{array}{c}\text { Grão de } \\
\text { soja }\end{array}$ & $\begin{array}{l}\text { Óleo de } \\
\text { soja }\end{array}$ & CV $(\%)$ \\
\hline $4: 00$ & 1,38 & 1,56 & 1,07 & 18,91 \\
\hline $6: 00$ & 1,47 & 1,51 & 1,15 & 24,52 \\
\hline 8:00 & $1,27 \mathrm{a}$ & $1,21 \mathrm{a}$ & $0,98 \mathrm{~b}$ & 26,82 \\
\hline $10: 00$ & 2,96 & 2,48 & 2,35 & 26,84 \\
\hline $12: 00$ & $3,37 \mathrm{a}$ & $2,96 a$ & $2,72 b$ & 25,46 \\
\hline 13:00 & 0,22 & 0,23 & 0,21 & 23,39 \\
\hline $14: 00$ & $12,06 \mathrm{a}$ & $11,70 \mathrm{a}$ & $10,83 b$ & 21,18 \\
\hline $14: 1 \omega 9$ & 0,30 & 0,29 & 0,35 & 16,67 \\
\hline $14: 1 \omega 7$ & 0,54 & 0,49 & 0,49 & 16,99 \\
\hline $14: 1 \omega 6$ & 0,67 & 0,52 & 0,50 & 17,82 \\
\hline $15: 00$ & 0,99 & 0,92 & 0,86 & 19,25 \\
\hline $15: 1 \omega 10$ & 0,41 & 0,39 & 0,39 & 16,64 \\
\hline $16: 00$ & $30,33 a$ & $29,23 b$ & $29,44 b$ & 16,50 \\
\hline $16: 1 \omega 9$ & $0,42 b$ & $0,42 b$ & $0,47 \mathrm{a}$ & 15,70 \\
\hline $16: 1 \omega 7$ & 1,37 & 1,16 & 1,16 & 13,53 \\
\hline $16: 1 \omega 5$ & 0,50 & 0,46 & 0,44 & 9,12 \\
\hline $17: 00$ & 0,54 & 0,54 & 0,49 & 19,41 \\
\hline $17: 1 \omega 9$ & 0,21 & 0,20 & 0,20 & 25,02 \\
\hline 18:00 & $14,89 \mathrm{a}$ & $14,73 a$ & $12,19 b$ & 14,32 \\
\hline $18: 1(11 t)$ & $1,88 \mathrm{~b}$ & $2,15 \mathrm{ab}$ & $3,00 \mathrm{a}$ & 27,34 \\
\hline $18: 1 \omega 9$ & $20,31 b$ & $21,46 b$ & $25,00 \mathrm{a}$ & 16,96 \\
\hline $18: 2 t$ & $0,30 \mathrm{ab}$ & $0,37 \mathrm{a}$ & $0,38 \mathrm{a}$ & 23,89 \\
\hline $18: 2 \omega 6$ & $1,56 \mathrm{~b}$ & $2,42 a$ & $1,88 \mathrm{~b}$ & 21,68 \\
\hline $20: 00$ & $0,12 b$ & $0,20 \mathrm{a}$ & $0,19 \mathrm{a}$ & 9,90 \\
\hline $18: 3 \omega 6$ & $0,25 \mathrm{c}$ & $0,30 \mathrm{~b}$ & $0,35 \mathrm{a}$ & 18,91 \\
\hline $18: 3 \omega 3$ & 0,32 & 0,51 & 0,42 & 24,52 \\
\hline CLA $[18: 2(9 c, 11 t)]$ & $0,98 \mathrm{c}$ & $1,11 \mathrm{~b}$ & $1,98 \mathrm{a}$ & 26,82 \\
\hline $22: 00$ & $0,19 b$ & $0,25 \mathrm{a}$ & $0,27 \mathrm{a}$ & 26,84 \\
\hline $22: 3 \omega 3$ & $0,20 \mathrm{~b}$ & $0,23 \mathrm{a}$ & $0,23 a$ & 25,46 \\
\hline AGPI & $3,60 \mathrm{~b}$ & $4,93 \mathrm{a}$ & $5,24 \mathrm{a}$ & 23,39 \\
\hline AGMI & $26,61 b$ & $27,55 b$ & $32,00 \mathrm{a}$ & 21,18 \\
\hline AGS & $69,78 b$ & $67,52 \mathrm{~b}$ & $62,76 \mathrm{a}$ & 16,67 \\
\hline$\omega-6$ & $2,47 b$ & $3,24 \mathrm{a}$ & $2,74 \mathrm{~b}$ & 16,99 \\
\hline$\omega-3$ & $0,52 b$ & $0,73 a$ & $0,65 \mathrm{ab}$ & 17,82 \\
\hline AGINS/AGS & $0,43 b$ & $0,48 \mathrm{~b}$ & $0,59 \mathrm{a}$ & 19,25 \\
\hline$\omega-6 / \omega-3$ & $4,75 \mathrm{a}$ & $4,43 \mathrm{a}$ & $4,22 \mathrm{~b}$ & 16,64 \\
\hline
\end{tabular}

Médias na mesma linha seguidas de letras distintas diferem entre si, pelo teste Tukey $(\mathrm{P}<0,05)$.

CLA: ácido linoleico conjugado; AGPI: ácidos graxos poli-insaturados; AGMI: ácidos graxos monoinsaturados; AGINS/AGS: relação ácidos graxos insaturados:ácidos graxos saturados.

Os teores de CLA e ácido vaccênico aumentaram com a inclusão de lipídeos $(\mathrm{P}<0,05)$, com efeito mais destacado para a dieta com óleo de soja. Esta proporcionou aumento de $275 \%$ da concentração de CLA, em relação à dieta sem lipídeo adicional. A diferença na concentração de CLA do grão de soja para aquela sem lipídeo adicional foi de $32 \%$. 
Tabela 5. Perfil de ácidos graxos da gordura do queijo muçarela de búfalas alimentadas com diferentes fontes de lipídeos na dieta (\%)

\begin{tabular}{|c|c|c|c|c|}
\hline Componente & Sem lipídeo adicional & $\begin{array}{c}\text { Grão de } \\
\text { soja }\end{array}$ & $\begin{array}{c}\begin{array}{c}\text { Óleo de } \\
\text { soja }\end{array} \\
\end{array}$ & $\mathrm{CV}(\%)$ \\
\hline 4:0 & 1,48 & 1,56 & 1,31 & 16,50 \\
\hline $6: 0$ & 1,33 & 1,45 & 1,03 & 22,70 \\
\hline $8: 0$ & $1,53 b$ & $1,56 \mathrm{~b}$ & $1,28 \mathrm{a}$ & 13,53 \\
\hline 10:0 & 2,98 & 3,02 & 2,43 & 9,12 \\
\hline 11:0 & 0,08 & 0,05 & 0,08 & 19,41 \\
\hline 12:0 & $3,54 a$ & $3,44 a$ & $2,96 \mathrm{~b}$ & 13,02 \\
\hline 13:0 & 0,24 & 0,23 & 0,19 & 27,32 \\
\hline 14:0 & $13,83 \mathrm{a}$ & $13,52 \mathrm{a}$ & $11,66 \mathrm{~b}$ & 16,34 \\
\hline $14: 1 \omega 9$ & 0,31 & 0,28 & 0,32 & 9,96 \\
\hline $14: 1 \omega 7$ & 0,5 & 0,51 & 0,44 & 23,89 \\
\hline 14:1 $\omega 6$ & 0,75 & 0,63 & 0,59 & 21,68 \\
\hline $15: 0$ & 1,01 & 0,94 & 0,87 & 9,90 \\
\hline $15: 1 \omega 10$ & 0,43 & 0,45 & 0,40 & 17,68 \\
\hline $16: 0$ & $30,82 \mathrm{a}$ & $28,91 \mathrm{~b}$ & $26,01 \mathrm{~b}$ & 17,23 \\
\hline $16: 1 \omega 10$ & 0,42 & 0,44 & 0,44 & 8,67 \\
\hline $16: 1 \omega 9$ & 0,42 & 0,49 & 0,25 & 21,74 \\
\hline $16: 1 \omega 7$ & 1,24 & 1,27 & 1,26 & 20,45 \\
\hline 17:0 & 0,43 & 0,42 & 0,39 & 23,95 \\
\hline $17: 1 \omega 9$ & $0,20 \mathrm{a}$ & $0,21 \mathrm{a}$ & $0,16 b$ & 18,23 \\
\hline 18:0 & $13,62 \mathrm{a}$ & $11,93 b$ & $11,85 b$ & 27,38 \\
\hline 18:1(11t) & $1,06 \mathrm{c}$ & $1,43 b$ & $2,65 \mathrm{a}$ & 25,61 \\
\hline 18:1 $\omega 9$ & $20,12 \mathrm{c}$ & $22,65 b$ & $26,15 \mathrm{a}$ & 27,90 \\
\hline $18: 2 \omega 6$ & $1,51 b$ & $1,99 \mathrm{~b}$ & $2,84 \mathrm{a}$ & 18,29 \\
\hline $18: 3 \omega 6$ & 0,22 & 0,21 & 0,23 & 17,20 \\
\hline $18: 3 \omega 3$ & $0,21 b$ & $0,27 a b$ & $0,34 \mathrm{a}$ & 23,79 \\
\hline CLA [18:2(9c,11t)] & $1,04 \mathrm{c}$ & $1,37 \mathrm{~b}$ & $2,86 \mathrm{a}$ & 17,68 \\
\hline $20: 4 \omega 6$ & $0,13 b$ & $0,15 \mathrm{~b}$ & $0,24 \mathrm{a}$ & 26,52 \\
\hline $22: 0$ & $0,17 \mathrm{bc}$ & $0,19 b$ & $0,25 \mathrm{a}$ & 14,09 \\
\hline AGPI & $2,90 \mathrm{~b}$ & $3,97 \mathrm{a}$ & $3,25 \mathrm{a}$ & 19,81 \\
\hline AGMI & $25,45 \mathrm{c}$ & $28,36 \mathrm{~b}$ & $32,66 \mathrm{a}$ & 23,29 \\
\hline AGS & $71,65 \mathrm{a}$ & $67,67 \mathrm{~b}$ & $64,09 \mathrm{~b}$ & 16,38 \\
\hline$\omega-6$ & $3,06 \mathrm{~b}$ & $3,83 \mathrm{a}$ & $3,05 b$ & 25,61 \\
\hline$\omega-3$ & $0,59 b$ & $0,77 \mathrm{a}$ & $0,79 a$ & 27,41 \\
\hline AGI/AGS & $0,40 \mathrm{c}$ & $0,48 b$ & $0,56 \mathrm{a}$ & 19,64 \\
\hline$\omega-6 / \omega-3$ & $5,19 \mathrm{a}$ & $4,97 \mathrm{a}$ & $3,86 \mathrm{~b}$ & 27,20 \\
\hline
\end{tabular}

Médias na mesma linha seguidas de letras distintas diferem entre si, pelo teste Tukey $(\mathrm{P}<0,05)$.

CLA: ácido linoleico conjugado; AGPI: ácidos graxos poli-insaturados; AGMI: ácidos graxos monoinsaturados; AGINS/AGS: relação ácidos graxos insaturados:ácidos graxos saturados.

Os maiores teores de CLA encontrados nas dietas com óleo de soja, em relação às dietas com grão de soja $(\mathrm{P}<0,05)$ podem ser explicados pela maior disponibilidade dos AGINS do óleo para a biohidrogenação ruminal (Oliveira et al., 2008).
Normalmente, quando se utilizam fontes de lipídeos na forma de grãos moídos, estes estão protegidos pela matriz dos grãos (Dhiman et al., 2000). 
Dhiman et al. (1999) verificaram que o processamento do leite em queijo não altera o teor de CLA, sugerindo que os queijos contendo altas concentrações de CLA só são produzidos a partir do leite que contenha também alto teor.

O consumo de CLA está relacionado a sua atividade anticarcinogênica para vários tipos de tumores (Bauman et al., 2004). Dessa maneira, $100 \mathrm{~g}$ de queijo muçarela produzidos com leite de búfalas que consumiram a dieta com óleo de soja contêm cerca de $25,27 \mathrm{~g}$ de gordura, $722 \mathrm{mg}$ de CLA e $134 \mathrm{mg}$ de ácido vaccênico. Considerando-se que $20 \%$ do ácido vaccênico ingerido é transformado em CLA pelo organismo humano (Palmquist, 2001), isso resultaria em $856 \mathrm{mg}$ de CLA, enquanto na muçarela proveniente das búfalas que receberam a dieta sem lipídeo adicional, essa ingestão seria de $216 \mathrm{mg}$ de CLA. Dados na literatura mostram que o consumo de $300 \mathrm{mg} / \mathrm{dia}$ de CLA resulta em redução de até $40 \%$ na chance de desenvolvimento de tumores de seio, de cólon e de útero (Ip e Pariza, 2005).

\section{CONCLUSÕES}

A inclusão de óleo de soja na dieta de búfalas alterou a composição do leite, aumentando o teor de gordura e alterando o perfil de ácidos graxos tanto do leite, quanto do queijo tipo muçarela, produzindo um produto de melhor qualidade. $\mathrm{O}$ uso do grão de soja ou do óleo de soja na dieta de búfalas em lactação elevou os teores de CLA no leite e, consequentemente, no queijo tipo muçarela, com maior intensidade de resposta para a dieta contendo óleo de soja.

\section{REFERÊNCIAS BIBLIOGRÁFICAS}

AMARAL, F.R.; CARVALHO, L.B.; SILVA, $\mathrm{N}$. et al. Composição e contagem de células somáticas em leite bubalino na região do Alto São Francisco, Minas Gerais, Brasil. Rev. Inst. Lat. Cândido Tostes, v.59. p.37-41, 2004.

AMARAL, F.R.; CARVALHO, L.B.; SILVA, N. et al. Qualidade do leite de búfalas: composição. Rev. Bras. Reprod. Anim., v.29, p.106-110, 2005.

ANDRIGHETTO, C.; JORGE, A.M.; GOMES, M.I.F.V. et al. Efeito da monensina sódica sobre a produção e composição do leite, a produção de mozarela e o escore da condição corporal de búfalas Murrah. Rev. Bras. Zootec., v.34, p.641649, 2005.

ANIMAL and vegetable fats and oils Preparation of methyl esters of fatty acids. Geneve: ISO, 1978. p.1-6. (Method ISO 5509).

BAUMAN, D.E.; GRIINARI, M.G. Nutritional regulation of milk fat synthesis. Annu. Rev Nutr., v.23, p.203-227, 2003.

BAUMAN, D.E.; PERFIELD, J.W.; LOCK, A.L. Effect of trans fatty acids on milk fat and their impact on human health. In: PROCEEDINGS SOUTHWEST NUTRITION \& MANAGEMENT CONFERENCE, 2004, Tempe, AZ. p.41-52. Online. Disponível em: $<$ http://animal.cals.arizona.edu/swnmc/2004/procee dings.php>. Acessado em: 3 ago. 2004.

BAUMGARD, L.H.; SANGSTER, J.K.; BAUMAN, D.E. Milk fat synthesis in dairy cows is progressively reduced by increasing supplemental amounts of Trans-10, cis-12 conjugated linoleic acid (CLA). J. Nutr., v.131, p.1764-1769, 2001.

DHIMAN, T.R.; HELMINK, E.D.; McMAHON, D.J. et al. Conjugated linoleic acid content of milk and cheese from cows fed extruded oilseeds. J. Dairy Sci., v.82, p.412-419, 1999.

DHIMAN, T.R.; SATTER, L.D.; PARIZA, M.W. et al. Conjugated linoleic acid (CLA) content of milk from cows offered diets rich in linoleic and linolenic acid. J. Dairy Sci., v.83, p.1016-1027, 2000.

DUBEY, P.C.; SUMAN, C.L.; SANYAL, M.K. et al. Factors affecting composition of milk of buffaloes. Indian J. Anim. Sci., v.67, p.802-804, 1997.

EIFERT, E.C.; LANA, R.P.; LANNA, D.P.D. et al. Perfil de ácidos graxos e teor de ácido linoleico conjugado no leite de vacas alimentadas com dietas com diferentes fontes de carboidratos, com ou sem óleo de soja suplementado. Rev. Bras. Zootec., v.35, p.1829-1837, 2006.

FARFAN, J.A. Alimentos que influenciam os níveis de colesterol no organismo. In: SEMINÁRIO "Colesterol": análise, ocorrência, redução em alimentos e implicações na saúde. Campinas: ITAL, 1996. p.35-44.

FOX, P.F.; LAW, J.; SWEENEY, P.L.H. et al. Biochemistry of cheese ripening. In: Fox, P.F. 
(Ed). Cheese: chemistry, physics and microbiology. 2.ed. New York: Chapman and Hall, 1993. p.389-438.

GAINES, W.L.; DAVIDSON, F.A. The effect of advance in lactation and gestation on mammary activity. J. Gen. Physiol., v.2, p.325-332, 1925.

GRIINARI, M.J.; CORL, B.A.; LACY, S.H. et al. Conjugadet linoleic acid is synthesized endogenously in lactating dairy cows by $\Delta^{9}$ desaturase. Am. J. Soc. Nutr. Sci., v.62, p.22852291, 2000.

GRUMMER, R.R. Effect of feed on the composition of milk fat. J. Dairy Sci., v.74, p.3244-3257, 1991.

IP, C.; PARIZA, M. CLA (Conjugated linoleic acid). In: Interpretative review of recent nutrition research. Disponível em: $<$ www.nationaldairycouncil.org $>$. Acessado em: 20 jan. 2005.

JIANG, J.; BJOERCK, L.; FONDÉN, J. et al. Occurrence of conjugated Cis-9 trans-11 Octadecadienoic acid in bovine milk: effects of feed and dietary. J. Dairy Sci., v.79, p.438-445, 1996.

MACEDO, M.P.; WECHSLER, F.S.; RAMOS, A.A. et al. Composição físico-química e produção do leite de búfalas da raça mediterrâneo no oeste do Estado de São Paulo. Rev. Bras. Zootec., v.30, suppl.1, p.1084-1088, 2001.

MERTENS, D.R. Predicting intake and digestibility using mathematical models of ruminal function. J. Anim. Sci., v.64, p.15481558, 1987.

NADER FILHO, A.; SCHOKEN-ITURRINO, R.P.; ROSSI JÚNIOR et al. Influência do teor de proteínas totais na acidez e $\mathrm{pH}$ do leite de búfala. Rev. Int. Lat. Cândido Tostes, v.39, p.25, 1984.

NUTRIENT requirements of dairy cattle. Washington, DC: National Academic Science, 2001.

OFFICIAL methods of analysis of the AOAC. 12.ed. Washington, DC: AOAC, 1990. 1094p.

OLIVEIRA, M.A.; REIS, R.B.; LADEIRA, M.M. Produção e composição do leite de vacas alimentadas com dietas com diferentes proporções de forragem e teores de lipídeos Arq. Bras. Med. Vet. Zootec., v.59, p.759-766, 2007.
OLIVEIRA, R.L.; LADEIRA, M.M.; BARBOSA, M.A.A.F. et al. Ácido linoleico conjugado e perfil de ácidos graxos no músculo e na capa de gordura de novilhos bubalinos alimentados com diferentes fontes de lipídios. Arq. Bras. Med. Vet. Zootec., v.60, p.169-178, 2008.

PALMQUIST, D.L.; JENKINS, T. C. Fat in lactation rations: review. J. Dairy Sci., v.63, p.114, 1980 .

PALMQUIST, D.L. Ruminal and endogenous synthesis of CLA in cows. Aust. J. Dairy Technol., v.56, p.134-137, 2001.

PATEL, R.S.; MISTRY, V.V; Physicochemical and structural properties of ultra filtered buffalo milk and milk powder. J. Dairy Sci., v.80, p.812817, 1997.

POLIDORI, F.; SGOIFO ROSSI, C.A.; SENATORE, E.M. et al. Effect of recombinant bovine somatotropin and calcium salts of longchain fatty acids on milk from Italian Buffalo. $J$. Dairy Sci., v.80, p.2137-2142, 1997.

RABELLO, T.M., VALADARES FILHO, S.C., COELHO DA SILVA, J.F. et al. Grão de soja moído na alimentação de vacas em lactação. I. Consumos, produção e composição do leite. Rev. Bras. Zootec., v.25, p.345-356, 1996.

SISTEMA de análises estatísticas - SAEG. Viçosa: UFV, 1997.

VAN NIEUWENHOVE; GONZÁLEZ, S.; PÉREZ CHAIA, A. Conjugated linoleic acid in buffalo (Bubalus bubalis) milk from Northwest Argentina. Milchwissenschaft, v.59 p.506-508, 2004.

VAN SOEST, P.J.; ROBERTSON, J.B.; LEWIS, B.A. et al. Methods for dietary fiber, and nonstarch polysaccharides in relation to animal nutrition. J. Dairy Sci., v.74, 35833597, 1991.

VARGAS, L.H.; LANA, R.P.; JHAM, G.N. et al. Adição de Lipídios na Ração de Vacas Leiteiras: Parâmetros Fermentativos Ruminais, Produção e Composição do Leite. Rev. Bras. Zootec., v.31, p.522-529, 2002.

VERRUMA, M.R.; SALGADO, J.M. Análise química do leite de búfala em comparação ao leite de vaca. Sci. Agric., v.51, p.131-137, 1994. 